\title{
RENORMALIZATION GROUP OF AND CONVERGENCE TO THE LISDLG PROCESS *
}

\author{
ENDRE IGLÓI ${ }^{1}$
}

\begin{abstract}
The LISDLG process denoted by $J(t)$ is defined in Iglói and Terdik [ESAIM: PS 7 (2003) 23-86] by a functional limit theorem as the limit of ISDLG processes. This paper gives a more general limit representation of $J(t)$. It is shown that process $J(t)$ has its own renormalization group and that $J(t)$ can be represented as the limit process of the renormalization operator flow applied to the elements of some set of stochastic processes. The latter set consists of IGSDLG processes which are generalizations of the ISDLG process.
\end{abstract}

Mathematics Subject Classification. 60F17, 60G10, 62M10.

Received January 15, 2003. Revised February 19, 2004.

\section{INTRODUCTION}

This paper can be considered to be the supplement of [3] insomuch as it is built upon the results of that paper. So the DLG, SDLG and LISDLG processes are supposed to be known to the reader.

The motivation of this paper is the following. It is well-known that the FBM (Fractional Brownian Motion) is a fixed point of its renormalization operators framing a group, or a fixed point of its renormalization group for short. With the help of the renormalization operator one can give functionals of appropriate processes which will converge to the FBM. There holds also a more general result of this type, coupling to a higher order generalization of the FBM, the Gaussian subordinated higher Hermite order self-similar process with stationary increments, see the classic papers [2] and [6]. The analog holds also for the LISDLG process and this is the subject of this paper.

In Section 2 we show that the LISDLG process has its own renormalization group and this fact gives an idea of what to do with certain proper processes to get the LISDLG process as a limit: apply the renormalization operator flow to the process and take the limit. Heuristically, if the initial process is not too far from the LISDLG process, the convergence must work. It works indeed, as we will see in Section 4. The main theorem of this paper, Theorem 4 states this convergence in more general circumstances. Namely, if the initial process is an element of the set of IGSDLG (Integrated General SDLG) processes, then the sums of an appropriate number of independent copies of it, after rescaling, will converge to the LISDLG process.

\footnotetext{
Keywords and phrases. LISDLG process, dilative stability, renormalization group, functional limit theorem, regularly varying function.

* This research is supported by the Hungarian National Science Foundation OTKA No. T 032658.

${ }^{1}$ Institute of Informatics, University of Debrecen, 4010 Debrecen, PF 12, Hungary; e-mail: igloi@inf .unideb.hu
} 
Section 3 is about GSDLG (General SDLG) processes. These are the processes from which we derive the above mentioned IGSDLG processes, by taking the integral and adjusting the mean to zero. The construction of a GSDLG process is similar to that of an SDLG process (see [3], Sect. 3.1), that is superposition of independent (conditionally on the sequence of random parameters $-2 \alpha_{k}$ ) stationary DLG processes with properly chosen random parameters. The random parameters $-2 \alpha_{k}$ have the same role as in the buildup of the SDLG process. However, due to the much milder conditions imposed on the sequence $\left\langle-2 \alpha_{k}\right\rangle_{k \in \mathbb{N}}$ in the GSDLG setup (ergodicity instead of independence, fairly general one dimensional distribution instead of the fixed $\Gamma)$, the resulting GSDLG processes constitute an entire class of processes instead of the single SDLG one.

In this paper all the stochastic processes are real-valued and the time parameter is nonnegative.

\section{The Renormalization Group of the LISDLG PROCESS}

Recall that the LISDLG process $\{J(t), t \geq 0\}$ can be defined as the a.s. (almost surerely) continuous process which has a zero mean and the following second and higher order joint cumulants (see [3], Prop. 14):

$$
\operatorname{cum}\left(J\left(t_{1}\right), \ldots, J\left(t_{m}\right)\right)=\frac{2^{1-2 H}}{1-H} \cdot \frac{c_{0}}{\sigma_{0}^{2}} \sigma_{0}^{2 m}(m-1) ! \operatorname{sym}_{\underline{t}}\left(\int_{0}^{\underline{t}} D_{\tau_{0}}(\underline{s})^{2(H-1)} \mathrm{d} \underline{s}\right)
$$

$m \geq 2$. Here $H \in\left(\frac{1}{2}, 1\right), c_{0}>0$ and $\sigma_{0}^{2}>0$ are constants, the operator sym is the symmetrization operator, symmetrization for all permutations of the components of $\underline{t}=\left(t_{1}, \ldots, \underline{t_{m}}\right)$, i.e. for any function $f(\underline{t})$ it is defined by

the expression

$$
\operatorname{sym}_{\underline{t}} f(\underline{t}) \doteq \frac{1}{m !} \sum_{\left(i_{1}, \ldots, i_{m}\right) \in \operatorname{Perm}(1,2, \ldots, m)} f\left(t_{i_{1}}, \ldots, t_{i_{m}}\right),
$$

$$
D_{\tau}(\underline{t}) \doteq\left|t_{i_{1}}-t_{1}\right|+\left|t_{i_{2}}-t_{i_{1}}\right|+\cdots+\left|t_{i_{m-1}}-t_{i_{m-2}}\right|+\left|t_{1}-t_{i_{m-1}}\right|,
$$

$m \in\{2,3,4, \ldots\}, \tau=\left(i_{1}, \ldots, i_{m-1}\right) \in \operatorname{Perm}(2,3, \ldots, m)$, and $\tau_{0}$ is the identity permutation. The LISDLG process has the remarkable property called dilative stability (see [3], Def. 3 and Th. 8), meaning that for every $T>0, m \in \mathbb{N}$ and $t_{1}, \ldots, t_{m} \geqslant 0$

$$
\left(\frac{J\left(T t_{1}\right)}{T}, \ldots, \frac{J\left(T t_{m}\right)}{T}\right)^{\circledast T^{2(1-H)}} \stackrel{\mathrm{d}}{\sim}\left(J\left(t_{1}\right), \ldots, J\left(t_{m}\right)\right)
$$

where $\stackrel{\mathrm{d}}{\sim}$ denotes equality in distribution and the exponent $\circledast T^{2(1-H)}$ denotes the $\left(T^{2(1-H)}\right)$ th convolution power.

In the remainder of this section we consider the processes in the weak sense, that is, we do not distinguish two processes if they are weakly equivalent, i.e. if all the corresponding finite dimensional distributions are the same.

Following [5] a stochastic process is called infinitely divisible, if its finite dimensional distributions are infinitely divisible. Denote the set of infinitely divisible processes by $\mathcal{I}$. In $\mathcal{I}$ for every $r>0$ we can define the operation called $r$ th convolution power and denote by ${ }^{\circledast r}$ (in the exponent). Let us define in $\mathcal{I}$ the operators $A_{T}, T>0$, by

$$
\begin{aligned}
A_{T} & : \mathcal{I} \longrightarrow \mathcal{I}, \quad X \mapsto A_{T} X, \\
\left(A_{T} X\right)(t) & \doteq \frac{1}{T}(X(T t))^{\circledast T^{2(1-H)}}, \quad t \geq 0 .
\end{aligned}
$$

Like the renormalization operator of the FBM, operator $A_{T}$ will be called a renormalization operator. The motivation for this appellation will be given in Theorem 1 . Consider the set $\mathcal{G} \doteq\left\{A_{T}: T>0\right\}$ equipped with the composition operation, i.e. $\left(A_{T} \circ A_{S}\right) X \doteq A_{T} A_{S} X, S, T>0, X \in \mathcal{I}$. Because $T^{2(1-H)}$ is a multiplicative 
function of $T$, we have $A_{T} \circ A_{S}=A_{T S}$. Hence $(\mathcal{G}, \circ)$ is a multiplicative one parameter group, which will be called the renormalization group of the LISDLG process.

With the above terminology the dilative stability of the LISDLG process can be reformulated as follows.

Theorem 1. The LISDLG process $\{J(t), t \geq 0\}$ is a fixed point of the elements of its renormalization group, or briefly, $\{J(t), t \geq 0\}$ is a fixed point of $\mathcal{G}$.

Theorem 1 gives an idea of what to do with a process to get the LISDLG process as a limit: apply the renormalization operator $A_{T}$ to the process and take the limit as $T \rightarrow \infty$. Heuristically, if the initial process is not too far from the LISDLG process, the convergence probably works. It works indeed, as we will see in Section 4 . But before that, we make a digression to the inverse (in some sense) of the problem. Let $\stackrel{\mathrm{fd}}{\longrightarrow}$ denote the convergence of processes in the sense of convergence of finite dimensional distributions.

Theorem 2. Let $\{X(t), t \geq 0\} \in \mathcal{I}$. If $\left\{A_{T} X(t), t \geq 0\right\} \underset{T \rightarrow \infty}{\stackrel{f d}{\longrightarrow}}\{Y(t), t \geq 0\}$, where $\{Y(t), t \geq 0\}$ is some (necessarily also infinitely divisible) process, then $\{Y(t), t \geq 0\}$ is a fixed point of the renormalization group $\mathcal{G}$.

Proof. It follows easily, since $(\mathcal{G}, \circ)$ is a multiplicative one parameter group.

\section{GSDLG PROCESSES}

Firts of all recall the notion and certain properties of the DLG process. (See [3], Sect. 2 for a more detailed discussion.) Let $\mu>0, \alpha \in \mathbb{R}, \sigma>0$ be parameters and

$$
\delta \doteq \frac{\mu}{\sigma^{2}}
$$

A diffusion process with a differential generator of the form

$$
(\mu+2 \alpha x) \frac{\partial}{\partial x}+\frac{1}{2} 4 \sigma^{2} x \frac{\partial^{2}}{\partial x^{2}}
$$

(linear drift and diffusion coefficients) is called a $\delta$-dimensional diffusion process with a linear differential generator, or DLG process, for short. Equivalently, the $\delta$-dimensional DLG process $\{R(t), t \geq 0\}$ is the unique strong solution of the diffusion equation

$$
\mathrm{d} R(t)=(\mu+2 \alpha R(t)) \mathrm{d} t+2 \sigma \sqrt{R(t)} \mathrm{d} B(t)
$$

where $\{B(t), t \geq 0\}$ is a standard BM (Brownian Motion). We assume that $\alpha<0$ and that $\{R(t), t \geq 0\}$ starts from its stationary one-dimensional distribution, i.e. we deal with stationary DLG processes. The onedimensional distribution of process $\{R(t), t \geq 0\}$ is $\Gamma$, namely

$$
R(t) \sim \Gamma\left(\frac{\delta}{2}, \frac{\sigma^{2}}{-\alpha}\right) .
$$

More generally, the $m$-dimensional distribution of process $\{R(t), t \geq 0\}$ is $m$-dimensional $\Gamma$, namely the characteristic function of the random vector $\left(R\left(t_{1}\right), \ldots, R\left(t_{m}\right)\right)$ is

$$
\varphi_{R\left(t_{1}\right), \ldots, R\left(t_{m}\right)}(\underline{u})=|I-2 i U \Sigma|^{-\delta / 2},
$$


where $U \doteq \operatorname{diag}(\underline{u})$ with $\underline{u} \in \mathbb{R}^{m}$, i.e. a matrix with zero elements except for the main diagonal, which is $\underline{u}$, $I$ is the $m \times m$ identity matrix, and $\mid$ denotes the determinant. Moreover, the cumulants are

$$
\operatorname{cum}\left(R\left(t_{1}\right), \ldots, R\left(t_{m}\right)\right)= \begin{cases}\frac{\delta}{2}\left(\frac{-\alpha}{\sigma^{2}}\right)^{-m}, & \text { if } m=1 \\ \frac{\delta}{2}\left(\frac{-\alpha}{\sigma^{2}}\right)^{-m} \sum_{\tau \in \operatorname{Perm}(2, \ldots, m)} \mathrm{e}^{\alpha D_{\tau}\left(t_{1}, \ldots, t_{m}\right)}, & \text { if } m \geqslant 2\end{cases}
$$

where $D_{\tau}\left(t_{1}, \ldots, t_{m}\right)$ was defined in $(2.2)$.

The construction of the GSDLG (General SDLG) process is similar to that of the SDLG process (see [3], Sect. 3.1), that is superposition of stationary DLG processes with properly chosen random parameters. Let $\langle\alpha\rangle=\left\langle\alpha_{k}\right\rangle_{k \in \mathbb{N}}$ be a stationary random sequence of positive parameters, and let $P_{\langle\alpha\rangle}$ denote its distribution on $\mathbb{R}_{+}^{\mathbb{N}}$. The other random parameters $\sigma_{k}^{2}, k \in \mathbb{N}$, depend on parameters $\alpha_{k}$ such that $\sigma_{k}^{2} \doteq-\alpha_{k} \sigma_{0}^{2}$, where $\sigma_{0}^{2}>0$ is a deterministic constant. The same notation will be used for the random sequence $\langle\alpha\rangle$ and for its realizations, i.e. $\langle\alpha\rangle$ may denote also an element of $\mathbb{R}_{+}^{\mathbb{N}}$; similarly, $\alpha_{k}$ and $\sigma_{k}^{2}$ can be both r.v.s (random variables) and fixed realizations. Furthermore let the deterministic parameters $\mu_{n} \doteq \mu_{0} / n, n \in \mathbb{N}$, where $\mu_{0}>0$ is a constant. Consider the triangular array

$$
\begin{array}{ccc}
R_{1,1}\left(t, \alpha_{1}\right) & & \\
R_{2,1}\left(t, \alpha_{1}\right), & R_{2,2}\left(t, \alpha_{2}\right) & \\
\vdots & & \\
R_{n, 1}\left(t, \alpha_{1}\right), & \ldots, & R_{n, n}\left(t, \alpha_{n}\right)
\end{array}
$$

$t \geq 0$, of stationary DLG processes $\left\{R_{n, k}\left(t, \alpha_{k}\right), t \geq 0\right\}$, where the second argument indicates the parameter $\alpha$ of the DLG process. Each process $\left\{R_{n, k}\left(t, \alpha_{k}\right), t \geq 0\right\}$ is defined for $P_{\langle\alpha\rangle}$-a.e. (almost every) $\langle\alpha\rangle$, with input BM $\left\{B_{k}(t), t \geq 0\right\}$ and parameters $\alpha_{k}, \sigma_{k}$, and $\mu_{n}$. Assume that the random sequence $\langle\alpha\rangle$ and the BMs are defined on two different probability spaces, $\left(\Omega_{A}, \mathcal{A}_{A}, P_{A}\right)$ and $\left(\Omega_{B}, \mathcal{A}_{B}, P_{B}\right)$, respectively ${ }^{1}$. Moreover, let the BMs $\left\{B_{k}(t), t \geq 0\right\}, k \in \mathbb{N}$, be independent. Thus, the following stochastic differential equations holding for $P_{\langle\alpha\rangle}$-a.e. $\langle\alpha\rangle$ link the processes and their parameters:

$$
\mathrm{d} R_{n, k}\left(t, \alpha_{k}\right)=\left(\mu_{n}+2 \alpha_{k} R_{n, k}\left(t, \alpha_{k}\right)\right) \mathrm{d} t+2 \sigma_{k} \sqrt{R_{n, k}\left(t, \alpha_{k}\right)} \mathrm{d} B_{k}(t)
$$

$k=1, \ldots, n ; n \in \mathbb{N}$.

We state that if in the above setup the distribution $P_{\langle\alpha\rangle}$ is chosen properly, then the row sums of (3.5) converge in a certain sense to a limit process $\{Y(t), t \geq 0\}$, which no longer depend on $\langle\alpha\rangle$, i.e.

$$
\left\{Z_{n}(t,\langle\alpha\rangle), t \geq 0\right\} \doteq\left\{\sum_{k=1}^{n} R_{n, k}\left(t, \alpha_{k}\right), t \geq 0\right\} \underset{n \rightarrow \infty}{\longrightarrow}\{Y(t), t \geq 0\}
$$

Process $\{Y(t), t \geq 0\}$ will be called a GSDLG process.

There are two differences between the base conditions of the two setups, the SDLG setup in [3], Section 3.1 and our GSDLG one. One is that the random parameters $\alpha_{k}, k=1,2, \ldots$, will not be assumed to be independent but only to constitute an ergodic sequence. The other difference - and this is more important - is that in the present setup the distribution of $-2 \alpha$ is not fully specified, resulting in an entire class of processes instead of a single process. Namely, in the construction of the SDLG process $-2 \alpha$ had $\Gamma(3-2 H, 1 / \lambda)$ distribution, where $H$

\footnotetext{
${ }^{1}$ The subscripts $A$ (large Greek alpha) and $B$ are used to remind of the parameter $\alpha$ and the BMs, respectively.
} 
and $\lambda$ were parameters. Hence the SDLG process could depend only on the parameters $H$ and $\lambda$ (besides, of course, $\mu_{0}$ and $\sigma_{0}^{2}$ ). The GSDLG process will, however, be more general as it will depend on the distribution of $-2 \alpha$, or, equivalently, on its d.f. $F_{-2 \alpha}$, and d.f. $F_{-2 \alpha}$ may be arbitrary in some class $\mathcal{F}$ of d.f.s. For this reason if necessary, we will also use the name $\operatorname{GSDLG}(F)$ to allude to $F \in \mathcal{F}$. The class $\mathcal{F}$ will, naturally, contain the $\Gamma(3-2 H, 1 / \lambda)$ distribution function. Accordingly, the class of GSDLG processes will contain the SDLG process. Now we summarize these conditions exactly.

Condition 1'. $1 / 2<H<1, \sigma_{0}^{2}>0$ and $\mu_{0}>0$ are constants.

Condition 2. $\left(\Omega_{A}, \mathcal{A}_{A}, P_{A}\right)$ and $\left(\Omega_{B}, \mathcal{A}_{B}, P_{B}\right)$ are two different probability spaces.

Condition 3'. a) $\langle\alpha\rangle=\left\langle\alpha_{k}\right\rangle_{k \in \mathbb{N}}$, is an ergodic sequence of r.v.s defined on $\left(\Omega_{A}, \mathcal{A}_{A}, P_{A}\right)$; its distribution on $R_{+}^{\mathbb{N}}$ is denoted by $P_{\langle\alpha\rangle}$.

b) The d.f. of the random parameters $-2 \alpha_{k}, F_{-2 \alpha} \in \mathcal{F}$, where

$$
\mathcal{F} \doteq\left\{d . f . F \text { regularly varying at zero of order } 3-2 H \text { and } \int_{0}^{\infty} x \mathrm{~d} F_{-2 \alpha}(x)<\infty\right\} .
$$

Condition 4. $\sigma_{k}^{2} \doteq-\alpha_{k} \sigma_{0}^{2}, k \in \mathbb{N}$.

Condition 5. $\mu_{n} \doteq \mu_{0} / n, n \in \mathbb{N}$.

Condition 6. $\left\{B_{k}(t), t \geq 0\right\}, k \in N$, are independent BMs defined on $\left(\Omega_{B}, \mathcal{A}_{B}, P_{B}\right)$.

Conditions 2, 4, 5, and 6 are the same as in [3], Section 3.1, and Condition 1' is almost the same as Condition 1 but without $\lambda$. Condition 3'b means that r.v.s $-2 \alpha_{k}$ are nonnegative, $\mathrm{E}_{A}\left(-2 \alpha_{k}\right)<\infty$, and $F_{-2 \alpha}$ is of the form

$$
F_{-2 \alpha}(x)=L_{0}(x) x^{3-2 H}, \quad x>0
$$

where the function $\left\{L_{0}(x), x>0\right\}$ is slowly varying in zero, i.e.

$$
\lim _{x \rightarrow 0} \frac{L_{0}(a x)}{L_{0}(x)}=1, \quad \forall a>0 .
$$

Remark 1. Condition 3'b is fulfilled when besides having a finite mean and restricted to the positive half-line, $F_{-2 \alpha}$ is absolutely continuous with a density function regularly varying in zero of order $2-2 H$; e.g. when the distribution of $-2 \alpha$ is $\Gamma$ with shape parameter $3-2 H$ (this is the SDLG case).

The following remark helps to size up how broad or narrow the set $\mathcal{F}$ is.

Remark 2. The intersection of $\mathcal{F}$ (which is here considered to be made up of distributions) with the set of positive selfdecomposable distributions is the set of shot noise distributions with exponential response functions. The latter distributions form a large class among the selfdecomposable distributions, see [4].

Mainly the law of large numbers relating to the following two cases will account for the existence of the GSDLG process.

Lemma 1. For $P_{\langle\alpha\rangle}$-a.e. $\langle\alpha\rangle$

$$
\lim _{n \rightarrow \infty}\left(\frac{1}{n} \sum_{k=1}^{n}\left(-2 \alpha_{k}\right)\right)=\mathrm{E}_{A}(-2 \alpha) \in \mathbb{R}_{+}
$$

and

$$
\lim _{n \rightarrow \infty}\left(\frac{1}{n} \sum_{k=1}^{n}\left(\frac{1}{-2 \alpha_{k}} \mathrm{e}^{2 \alpha_{k} x}\right)\right)=\mathrm{E}_{A}\left(\frac{1}{-2 \alpha} \mathrm{e}^{2 \alpha x}\right) \in \mathbb{R}_{+},
$$

for every $x \geq 0$. 
Proof. The first statement is a consequence of Conditions 3'a and 3'b $\left(\mathrm{E}_{A}(-2 \alpha)<\infty\right)$. To prove that the right hand side of (3.9) is finite, it is enough to show that

$$
\int_{0}^{1} \frac{1}{y} \mathrm{~d} F_{-2 \alpha}(y)<\infty
$$

Now, let $0<\varepsilon<1$. Integrating by parts and using (3.7) we have

$$
\int_{\varepsilon}^{1} \frac{1}{y} \mathrm{~d} F_{-2 \alpha}(y)=L_{0}(1)-L_{0}(\varepsilon) \varepsilon^{2-2 H}+\int_{\varepsilon}^{1} L_{0}(y) y^{1-2 H} \mathrm{~d} y .
$$

Since by the first part of Condition 1' there exists $\delta>0$ such that $\lim _{y \downarrow 0}\left(L_{0}(y) y^{1-2 H+1-\delta}\right)=0$, we have $L_{0}(y) y^{1-2 H} \in L^{1}[0,1]$. Letting $\varepsilon \rightarrow 0$ in (3.11) we obtain the finiteness of the right hand side and thus of the left hand side as well. Application of the monotone convergence theorem yields (3.10). Finally, Condition 3'a ensures the convergence in (3.9).

To stress it again, we have the stationary DLG processes $\left\{R_{n, k}\left(t, \alpha_{k}\right), t \geq 0\right\}$ defined for $P_{\langle\alpha\rangle}$-a.e. $\langle\alpha\rangle$ by (3.6). Specifically, $\left\{R_{1,1}\left(t, \alpha_{1}\right), t \geq 0\right\}$ has (random) parameters $\alpha_{1}, \sigma_{1}^{2}=-\alpha_{1} \sigma_{0}^{2}$ and (non-random) $\mu_{1}=\mu_{0}$. Due to (3.2) for any fixed $t$ and for $P_{\langle\alpha\rangle}$-a.e. $\langle\alpha\rangle$ the distribution of $R_{n, k}\left(t, \alpha_{k}\right)$ (the conditional distribution of $R_{n, k}\left(t, \alpha_{k}\right)$, given $\left.\langle\alpha\rangle\right)$ is $\Gamma\left(\delta_{n, k} / 2,-\sigma_{k}^{2} / \alpha_{k}\right)$, where $\delta_{n, k}=\mu_{n} / \sigma_{k}^{2}$, i.e.

$$
R_{n, k}\left(t, \alpha_{k}\right) \sim \Gamma\left(\frac{\mu_{0}}{2 n \sigma_{k}^{2}}, \sigma_{0}^{2}\right) \sim \Gamma\left(\frac{\mu_{0}}{\sigma_{0}^{2} n} \frac{1}{-2 \alpha_{k}}, \sigma_{0}^{2}\right)
$$

for $P_{\langle\alpha\rangle}$-a.e. $\langle\alpha\rangle$.

Let us define the process $\left\{Z_{n}(t,\langle\alpha\rangle), t \geq 0\right\}$ to be the $n$th row sum of the triangular array (3.5), i.e.

$$
Z_{n}(t,\langle\alpha\rangle) \doteq \sum_{k=1}^{n} R_{n, k}\left(t, \alpha_{k}\right)
$$

$t \geq 0$, for $P_{\langle\alpha\rangle}$-a.e. $\langle\alpha\rangle, n \in \mathbb{N}$. The first observation is that for $P_{\langle\alpha\rangle}$-a.e. $\langle\alpha\rangle$ the one-dimensional limit distribution of $\left\{Z_{n}(t,\langle\alpha\rangle), t \geq 0\right\}$ remains $\Gamma$ as $n \rightarrow \infty$.

Proposition 1. For every fixed $t \in \mathbb{R}_{+}$and for $P_{\langle\alpha\rangle}$-a.e. $\langle\alpha\rangle$ the distribution of $Z_{n}(t,\langle\alpha\rangle)$ (the conditional distribution of $Z_{n}(t,\langle\alpha\rangle)$, given $\left.\langle\alpha\rangle\right)$ converges to a $\Gamma$ distribution, namely

$$
Z_{n}(t,\langle\alpha\rangle) \underset{n \rightarrow \infty}{\stackrel{w}{\longrightarrow}} \Gamma\left(\frac{\mu_{0}}{\sigma_{0}^{2}} \mathrm{E}_{A} \frac{1}{-2 \alpha}, \sigma_{0}^{2}\right)
$$

for $P_{\langle\alpha\rangle}$-a.e. $\langle\alpha\rangle$.

Proof. Because processes $\left\{R_{n, k}\left(t, \alpha_{k}\right), t \geq 0\right\}, k=1, \ldots, n$, are independent conditionally on $\langle\alpha\rangle$ (due to Condition 6$)$, by (3.12) and Condition 4 we have

$$
Z_{n}(t,\langle\alpha\rangle) \sim \Gamma\left(\frac{\mu_{0}}{n \sigma_{0}^{2}} \sum_{k=1}^{n} \frac{1}{-2 \alpha_{k}}, \sigma_{0}^{2}\right)
$$

for $P_{\langle\alpha\rangle}$-a.e. $\langle\alpha\rangle$, from which we have the statement by (3.9). 
Corollary 1. The GSDLG process $\{Y(t), t \geq 0\}$, if it exists, has marginal distribution $\Gamma$, namely,

$$
Y(t) \sim \Gamma\left(\frac{\mu_{0}}{\sigma_{0}^{2}} \mathrm{E}_{A} \frac{1}{-2 \alpha}, \sigma_{0}^{2}\right),
$$

for every $t \geqslant 0$.

Remark 3. For each $n \in \mathbb{N}$ and for $P_{\langle\alpha\rangle}$-a.e. $\langle\alpha\rangle$ the random process $\left\{Z_{n}(t,\langle\alpha\rangle), t \geq 0\right\}$ is $P_{B}$-a.s. continuous, i.e. for $P_{\langle\alpha\rangle}$-a.e. $\langle\alpha\rangle:\left\{Z_{n}(t,\langle\alpha\rangle), t \geq 0\right\} \in \mathcal{C}[0, \infty) P_{B}$-a.s. Thus, for each $n \in \mathbb{N}$ and for $P_{\langle\alpha\rangle}$-a.e. $\langle\alpha\rangle$ : process $\left\{Z_{n}(t,\langle\alpha\rangle), t \geq 0\right\}$ induces a measure $P_{Z_{n,\langle\alpha\rangle}}$ on $\mathcal{C}[0, \infty)$.

Before stating the main theorem in this section we need the following lemma, which is point by point the same as Lemma 3 in [3].

Lemma 2. For $P_{\langle\alpha\rangle}$-a.e. $\langle\alpha\rangle$ the sequence of measures $P_{Z_{n,\langle\alpha\rangle}}, n \in \mathbb{N}$, mentioned in Remark 3, is tight.

Proof. We premise that in this proof every statement containing realizations of $\langle\alpha\rangle$, will be meant to hold for $P_{\langle\alpha\rangle}$-a.e. $\langle\alpha\rangle$.

It follows from (3.3) that the characteristic function of $R_{n, k}\left(t, \alpha_{k}\right)-R_{n, k}\left(0, \alpha_{k}\right)$ is

$$
\begin{aligned}
\varphi_{R_{n, k}\left(t, \alpha_{k}\right)-R_{n, k}\left(0, \alpha_{k}\right)}(u) & =\varphi_{R_{n, k}\left(t, \alpha_{k}\right), R_{n, k}\left(0, \alpha_{k}\right)}(u,-u) \\
& =\left|I-i u \sigma_{0}^{2}\left(\begin{array}{cc}
1 & 0 \\
0 & -1
\end{array}\right)\left(\begin{array}{cc}
1 & \mathrm{e}^{\alpha_{k}|t|} \\
\mathrm{e}^{\alpha_{k}|t|} & 1
\end{array}\right)\right|^{-\frac{\mu_{0}}{-2 n \sigma_{0}^{2} \alpha_{k}}} \\
& =\left(1+u^{2} \sigma_{0}^{4}\left(1-\mathrm{e}^{2 \alpha_{k}|t|}\right)\right)^{-\frac{\mu_{0}}{-2 n \sigma_{0}^{2} \alpha_{k}}}
\end{aligned}
$$

Calculating the moments from the characteristic function by differentiation, we have

$$
\begin{aligned}
& \mathrm{E}\left(R_{n, k}\left(t, \alpha_{k}\right)-R_{n, k}\left(0, \alpha_{k}\right)\right)^{2}=\frac{2 \mu_{0} \sigma_{0}^{2}}{n} \cdot \frac{1-\mathrm{e}^{2 \alpha_{k}|t|}}{-2 \alpha_{k}} \\
& \mathrm{E}\left(R_{n, k}\left(t, \alpha_{k}\right)-R_{n, k}\left(0, \alpha_{k}\right)\right)^{4}=12 \frac{\mu_{0}}{-2 n \sigma_{0}^{2} \alpha_{k}}\left(\frac{\mu_{0}}{-2 n \sigma_{0}^{2} \alpha_{k}}+1\right)\left(\left(1-\mathrm{e}^{2 \alpha_{k}|t|}\right) \sigma_{0}^{4}\right)^{2} .
\end{aligned}
$$

Hence

$$
\begin{aligned}
\mathrm{E}\left(Z_{n}(t,\langle\alpha\rangle)-Z_{n}(0,\langle\alpha\rangle)\right)^{4}= & \sum_{k=1}^{n} \mathrm{E}\left(R_{n, k}\left(t, \alpha_{k}\right)-R_{n, k}\left(0, \alpha_{k}\right)\right)^{4} \\
& +\sum_{k=1}^{n} \sum_{\ell=1}^{n} \mathrm{E}\left(R_{n, k}\left(t, \alpha_{k}\right)-R_{n, k}\left(0, \alpha_{k}\right)\right)^{2} \mathrm{E}\left(R_{n, \ell}\left(t, \alpha_{\ell}\right)-R_{n, \ell}\left(0, \alpha_{\ell}\right)\right)^{2} \\
\leqslant & \left(12 \frac{\mu_{0} \sigma_{0}^{6}}{n} \sum_{k=1}^{n}\left(\frac{\mu_{0}}{\sigma_{0}^{2} n}-2 \alpha_{k}\right)+\frac{4 \mu_{0}^{2} \sigma_{0}^{4}}{n^{2}} \sum_{k=1}^{n} \sum_{\ell=1}^{n} 1\right) t^{2} \\
= & \left(4 \mu_{0}^{2} \sigma_{0}^{4}\left(1+\frac{3}{n}\right)+\frac{12 \mu_{0} \sigma_{0}^{6}}{n} \sum_{k=1}^{n}\left(-2 \alpha_{k}\right)\right) t^{2} \\
\leqslant & \left(16 \mu_{0}^{2} \sigma_{0}^{4}+12 \mu_{0} \sigma_{0}^{6} K(\langle\alpha\rangle)\right) t^{2}
\end{aligned}
$$

for all $n \in \mathbb{N}$, where $K(\langle\alpha\rangle)$ is a constant bounding the series $\frac{1}{n} \sum_{k=1}^{n}\left(-2 \alpha_{k}\right)$ from above. Such a constant exists by the strong law of large numbers. 
Process $\left\{Z_{n}(t,\langle\alpha\rangle), t \geq 0\right\}$ is stationary, thus it has stationary increments. Consequently, using (3.13), we have

$$
\mathrm{E}\left(Z_{n}(t+s,\langle\alpha\rangle)-Z_{n}(s,\langle\alpha\rangle)\right)^{4} \leqslant K_{1}(\langle\alpha\rangle) t^{2},
$$

for all $t, s>0$ and $n \in \mathbb{N}$. Thus the tightness condition of Kolmogorov is fulfilled.

In the rest of the section we lay down the theorem about the existence of the GSDLG process (corresponding partly to Th. 5 in [3]). Process $\{Y(t), t \geq 0\}$ of Theorem 3 is what we call GSDLG process.

Theorem 3. Let Conditions 1', 2, 3, 4, 5, 6 hold. Then for $P_{\langle\alpha\rangle}$-a.e. $\langle\alpha\rangle$ the series of processes

$$
Z_{n}(t,\langle\alpha\rangle) \doteq \sum_{k=1}^{n} R_{n, k}\left(t, \alpha_{k}\right)
$$

$t \geq 0$, converges weakly to some a.s. continuous process $\left\{Y_{F}(t), t \geq 0\right\}$ defined on $\left(\Omega_{B}, \mathcal{A}_{B}, P_{B}\right)$, i.e.

$$
\left\{Z_{n}(t,\langle\alpha\rangle), t \geq 0\right\} \underset{n \rightarrow \infty}{\stackrel{w}{\longrightarrow}}\{Y(t), t \geq 0\} \quad \text { in } \mathcal{C}[0, \infty)
$$

for $P_{\langle\alpha\rangle}$-a.e. $\langle\alpha\rangle$. Process $\{Y(t), t \geq 0\}$ does not depend on the realizations $\langle\alpha\rangle$ any more, however it may depend on d.f. $F=F_{-2 \alpha} \in \mathcal{F}$ (hence we will write $Y_{F}(t)$ if needed). Moreover, its finite-dimensional distributions have the following cumulants:

$$
\operatorname{cum}\left(Y\left(t_{1}\right), \ldots, Y\left(t_{m}\right)\right)=\left\{\begin{array}{ll}
\mu_{0} \sigma_{0}^{2 m-2} \sum_{\tau \in \operatorname{Perm}(2, \ldots, m)} \mathrm{E}_{A}\left(\frac{1}{-2 \alpha} \mathrm{e}^{\alpha D_{\tau}(\underline{t})}\right) & \text { if } m \geq 2 \\
\mu_{0} \mathrm{E}_{A} \frac{1}{-2 \alpha} & \text { if } m=1
\end{array} .\right.
$$

Proof. We premise that in this proof every statement containing realizations of $\langle\alpha\rangle$, will be meant to hold for $P_{\langle\alpha\rangle}$-a.e. $\langle\alpha\rangle$.

Because processes $\left\{R_{n, k}\left(t, \alpha_{k}\right), t \geq 0\right\}, k=1, \ldots, n$, are independent, we have by (3.4), Conditions 4 and 5 , formula (3.1) and formula (3.9)

$$
\begin{aligned}
& \operatorname{cum}\left(Z_{n}\left(t_{1},\langle\alpha\rangle\right), \ldots, Z_{n}\left(t_{m},\langle\alpha\rangle\right)\right)=\sum_{k=1}^{n} \operatorname{cum}\left(R_{n, k}\left(t_{1}, \alpha_{k}\right), \ldots, R_{n, k}\left(t_{m}, \alpha_{k}\right)\right) \\
&=\mu_{0} \sigma_{0}^{2 m-2} \sum_{\tau \in \operatorname{Perm}(2, \ldots, m)} \frac{1}{n} \sum_{k=1}^{n}\left(\frac{1}{-2 \alpha_{k}} \mathrm{e}^{\alpha_{k} D_{\tau}(\underline{\underline{t}})}\right) \underset{n \rightarrow \infty}{\longrightarrow} \mu_{0} \sigma_{0}^{2 m-2} \sum_{\tau \in \operatorname{Perm}(2, \ldots, m)} \mathrm{E}_{A}\left(\frac{1}{-2 \alpha} \mathrm{e}^{\alpha D_{\tau}(\underline{\underline{t}})}\right),
\end{aligned}
$$

for $m \geq 2$. Taking the case $m=1$ similarly we have shown that $\operatorname{cum}\left(Z_{n}\left(t_{1},\langle\alpha\rangle\right), \ldots, Z_{n}\left(t_{m},\langle\alpha\rangle\right)\right)$ converges as $n \rightarrow \infty$ to the right hand side of (3.14). Next we prove that the cumulants on the right hand side of (3.14) uniquely determine a distribution on $\mathcal{C}[0, \infty)$. Since the sequence of measures $P_{Z_{n,\langle\alpha\rangle}}, n \in \mathbb{N}$, mentioned in Lemma 2 is tight, there exists a $P_{B}$-a.s. continuous process the joint cumulants of which are the ones on the right hand side of (3.14). Let $\{Y(t), t \geq 0\}$ be such a process. What we have to prove yet is the uniqueness, i.e. that the finite dimensional distributions of process $\{Y(t), t \geq 0\}$ are uniquely determined by the cumulants on the right hand side of (3.14). For this it is enough to prove that for an arbitrary linear combination

$$
\sum_{k=1}^{m} \lambda_{k} Y\left(t_{k}\right)
$$


its distribution is uniquely determined by its moments. But this is so because (3.15) does have a moment generating function, because each of the r.v.s $\lambda_{k} Y\left(t_{k}\right)$ has one (as they are $\Gamma$ distributed by Cor. 1 ), and by the Hölder inequality we have

$$
\mathrm{E} \exp \left(u \sum_{k=1}^{m} \lambda_{k} Y\left(t_{k}\right)\right) \leq \prod_{k=1}^{m}\left(\mathrm{E} \exp \left(m u \lambda_{k} Y\left(t_{k}\right)\right)\right)^{\frac{1}{m}}
$$

for arguments $u$ with sufficiently small modulus.

\section{Convergence of Rescaled sums of independent IGSDLG PRocesses TO THE LISDLG PROCESS}

Let $\left\{Y_{F}(t), t \geq 0\right\}$ be a GSDLG $(F)$ process. Its integral process with a mean adjusted to zero,

$$
\left\{J_{F}(t), t \geq 0\right\} \doteq\left\{\int_{0}^{t}\left(Y_{F}(s)-\mathrm{E} Y_{F}\right) \mathrm{d} s, t \geq 0\right\}
$$

will be called an IGSDLG $(F)$ process (Integrated GSDLG) ${ }^{2}$. The essence of this section is that the set of IGSDLG processes, i.e.

$$
\left\{\left\{J_{F}(t), t \geq 0\right\}: F \in \mathcal{F}\right\}
$$

is contained in the domain of attraction of the LISDLG process. We will state a functional limit theorem that leads from any IGSDLG process to the LISDLG process. Namely, the sums of an appropriate number of independent copies of an IGSDLG process, after rescaling, will converge to the LISDLG process. The convergence means the weak convergence of probability distributions on the space of continuous functions (see Th. 4). The "appropriate number" depends on the asymptotic behaviour in zero of the d.f. $F \in \mathcal{F}$. When $F$ is the $\Gamma(3-2 H, 1 / \lambda)$ d.f. (as in the SDLG case), the theorem merely states that when we apply the renormalization operator $A_{T}$ to the IGSDLG $(F)$ process, the resulting process converges weakly to the LISDLG process.

For shortness, introduce the following notation.

Notation 1. $L_{\infty}(x) \doteq L_{0}(1 / x), x>0$ (see (3.7) for $L_{0}(x)$ ).

The function $L_{\infty}(x)$ is slowly varying in infinity, i.e.

$$
\lim _{x \rightarrow \infty} \frac{L_{\infty}(a x)}{L_{\infty}(x)}=1, \quad \forall a>0,
$$

see (3.8). The next lemma is of a technical nature.

Lemma 3. For any $r>0$ we have

$$
\lim _{T \rightarrow \infty} E_{A}\left(\frac{1}{-2 \alpha} \mathrm{e}^{\alpha T r}\right) \frac{T^{2(1-H)}}{L_{\infty}(T)}=2^{2(1-H)}(3-2 H) \Gamma(2-2 H) r^{2(H-1)}
$$

and for all $0<\delta<2(1-H)$

$$
E_{A}\left(\frac{1}{-2 \alpha} \mathrm{e}^{\alpha T r}\right) \frac{T^{2(1-H)}}{L_{\infty}(T)} \leq k_{1} r^{2(H-1)+\delta}+k_{2} r^{2(H-1)-\delta},
$$

where $0<k_{1}, k_{2}$ are some constants (possibly depending on $\delta$ and $H$ ).

\footnotetext{
${ }^{2}$ In the notation $J_{F}(t)$ the index $F$ is always provided, because $J(t)$ without an index denotes the LISDLG process.
} 
Proof. By the form of the d.f. $F(x)=F_{-2 \alpha}(x)$ according to (3.7), after integrating by parts we have

$$
\mathrm{E}_{A}\left(\frac{1}{-2 \alpha} \mathrm{e}^{\alpha T r}\right) \frac{T^{2(1-H)}}{L_{\infty}(T)}=\int_{0}^{\infty} \frac{1}{x} \mathrm{e}^{-x T \frac{r}{2}} \mathrm{~d} F(x) \frac{T^{2(1-H)}}{L_{\infty}(T)}=\int_{0}^{\infty} x^{2-2 H}\left(\frac{1}{x}+1\right) \mathrm{e}^{-x} \frac{L_{\infty}\left(\frac{r}{2 x} T\right)}{L_{\infty}(T)} \mathrm{d} x\left(\frac{r}{2}\right)^{2(H-1)} .
$$

We may use Lebesgue's dominated convergence theorem, because on the one hand

$$
\lim _{T \rightarrow \infty}\left(x^{2-2 H}\left(\frac{1}{x}+1\right) \mathrm{e}^{-x} \frac{L_{\infty}\left(\frac{r}{2 x} T\right)}{L_{\infty}(T)}\right)=x^{2-2 H}\left(\frac{1}{x}+1\right) \mathrm{e}^{-x}
$$

for all $x>0$, since $L_{\infty}$ is slowly varying. On the other hand, the majorizing argument is the following. By a theorem of Potter (see [1], p. 25, Th. 1.5.6. (ii)) for every $\delta>0$ there exists a $k>0$ such that

$$
\frac{L_{\infty}\left(\frac{r}{2 x} T\right)}{L_{\infty}(T)} \leq k \max \left(\left(\frac{r}{2 x}\right)^{\delta},\left(\frac{r}{2 x}\right)^{-\delta}\right)
$$

for all $T>0$ and $x>0$. Therefore we have

$$
\begin{aligned}
x^{2-2 H}\left(\frac{1}{x}+1\right) \mathrm{e}^{-x} \frac{L_{\infty}\left(\frac{r}{2 x} T\right)}{L_{\infty}(T)} \leq & k x^{2-2 H}\left(\frac{1}{x}+1\right) \mathrm{e}^{-x} \max \left(\left(\frac{r}{2 x}\right)^{\delta},\left(\frac{r}{2 x}\right)^{-\delta}\right) \\
= & k \max \left(\left(\frac{r}{2}\right)^{\delta} x^{1-2 H-\delta},\left(\frac{r}{2}\right)^{-\delta} x^{1-2 H+\delta}\right) \mathrm{e}^{-x} \\
& +k \max \left(\left(\frac{r}{2}\right)^{\delta} x^{2-2 H-\delta},\left(\frac{r}{2}\right)^{-\delta} x^{2-2 H+\delta}\right) \mathrm{e}^{-x}
\end{aligned}
$$

On the right hand side of (4.4) in all of the four places the power of $x$ is greater than -1 if we choose $\delta$ so that $1-2 H-\delta>-1$, i.e. $0<\delta<2(1-H)$. Thus if $0<\delta<2(1-H)$, then the right hand side of (4.4) (as a function of $x$ ) is integrable and this fact means that the condition of the dominated convergence theorem is fulfilled. So by (4.2) and (4.3) the first statement of the lemma will follow if we calculate the integral

$$
\int_{0}^{\infty} \lim _{T \rightarrow \infty}\left(x^{2-2 H}\left(\frac{1}{x}+1\right) \mathrm{e}^{-x} \frac{L_{\infty}\left(\frac{r}{2 x} T\right)}{L_{\infty}(T)}\right) \mathrm{d} x=\int_{0}^{\infty} x^{2-2 H}\left(\frac{1}{x}+1\right) \mathrm{e}^{-x} \mathrm{~d} x=(3-2 H) \Gamma(2-2 H) .
$$

With respect to the second statement, using (4.2) and (4.4) we have

$$
\begin{aligned}
\mathrm{E}_{A}\left(\frac{1}{-2 \alpha} \mathrm{e}^{\alpha T r}\right) \frac{T^{2(1-H)}}{L_{\infty}(T)} \leq & 2^{2(1-H)} r^{2(H-1)} k\left\{\int_{0}^{\infty} \max \left(\left(\frac{r}{2}\right)^{\delta} x^{1-2 H-\delta},\left(\frac{r}{2}\right)^{-\delta} x^{1-2 H+\delta}\right) \mathrm{e}^{-x} \mathrm{~d} x\right. \\
& \left.+\int_{0}^{\infty} \max \left(\left(\frac{r}{2}\right)^{\delta} x^{2-2 H-\delta},\left(\frac{r}{2}\right)^{-\delta} x^{1-2 H+\delta}\right) \mathrm{e}^{-x} \mathrm{~d} x\right\} \\
\leq & 2^{2(1-H)} r^{2(H-1)} k\left\{\left(\frac{r}{2}\right)^{\delta} \Gamma(2-2 H-\delta)+\left(\frac{r}{2}\right)^{-\delta} \Gamma(2-2 H+\delta)\right. \\
& \left.+\left(\frac{r}{2}\right)^{\delta} \Gamma(3-2 H-\delta)+\left(\frac{r}{2}\right)^{-\delta} \Gamma(3-2 H+\delta)\right\} \\
= & k_{1} r^{2(H-1)+\delta}+k_{2} r^{2(H-1)-\delta},
\end{aligned}
$$

if $2-2 H-\delta>0$, i.e. $\delta<2(1-H)$. 
The next proposition already contains the key tool that will lead to our aim.

Proposition 2. Let $\left\{J_{F}(t), t \geq 0\right\}$ be an $I G S D L G(F)$ process and $m \geq 2,\left(t_{1}, \ldots, t_{m}\right) \in \mathbb{R}_{+}^{m}$. Then we have

$$
\begin{aligned}
& \operatorname{cum}\left(J_{F}\left(T t_{1}\right), \ldots, J_{F}\left(T t_{m}\right)\right) \underset{T \rightarrow \infty}{\sim} \mu_{0} \sigma_{0}^{2 m-2} 2^{2(1-H)}(3-2 H) \Gamma(2-2 H)(m-1) ! \\
& \times \operatorname{sym}_{\underline{t}}\left(\int_{0}^{t_{1}} \ldots \int_{0}^{t_{m}} D_{\tau_{0}}(\underline{s})^{2(H-1)} \mathrm{d} \underline{s}\right) T^{m+2(H-1)} L_{\infty}(T) .
\end{aligned}
$$

Proof. By (3.14) we have

$$
\begin{aligned}
\operatorname{cum}\left(J_{F}\left(T t_{1}\right), \ldots, J_{F}\left(T t_{m}\right)\right) & =\mu_{0} \sigma_{0}^{2 m-2} \sum_{\tau \in \operatorname{Perm}(2, \ldots, m)} \int_{0}^{T t_{1}} \cdots \int_{0}^{T t_{m}} \mathrm{E}_{A}\left(\frac{1}{-2 \alpha} \mathrm{e}^{\alpha D_{\tau}(\underline{s})}\right) \mathrm{d} \underline{s} \\
& =\mu_{0} \sigma_{0}^{2 m-2}(m-1) ! \operatorname{sym}_{\underline{t}}\left(\int_{0}^{t_{1}} \ldots \int_{0}^{t_{m}} \mathrm{E}_{A}\left(\frac{1}{-2 \alpha} \mathrm{e}^{\alpha T D_{\tau_{0}}(\underline{s})}\right) \mathrm{d} \underline{s}\right) T^{m}
\end{aligned}
$$

and from this, by Lemma 3 and Lebesgue's dominated convergence theorem we have the statement.

It seems from the proof of Proposition 2 that all IGSDLG processes are infinitely divisible, i.e. they are contained in $\mathcal{I}$. Hence it is meaningful to renormalize an IGSDLG process, i.e. to take the process $\left\{\left(A_{T} J_{F}\right)(t), t \geq 0\right\}$, or more generally, the process $\left\{\left(J_{F}(t)\right)^{\circledast a}, t \geq 0\right\}$ for any $a>0$. The following proposition is a direct consequence of Proposition 2.

Proposition 3. Let $\left\{J_{F}(t), t \geq 0\right\}$ be an $I G S D L G(F)$ process and $m \geq 2,\left(t_{1}, \ldots, t_{m}\right) \in \mathbb{R}_{+}^{m}$. Then we have

$$
\begin{aligned}
\lim _{T \rightarrow \infty} \operatorname{cum}\left(\left(\frac{1}{T} J_{F}\left(T t_{1}\right)\right)^{\circledast \frac{T^{2(1-H)}}{L_{\infty}(T)}}, \ldots,\left(\frac{1}{T} J_{F}\left(T t_{m}\right)\right)^{\circledast \frac{T^{2(1-H)}}{L_{\infty}(T)}}\right) \\
=\mu_{0} \sigma_{0}^{2 m-2} 2^{2(1-H)}(3-2 H) \Gamma(2-2 H)(m-1) ! \operatorname{sym}_{\underline{t}}\left(\int_{0}^{t_{1}} \ldots \int_{0}^{t_{m}} D_{\tau_{0}}(\underline{s})^{2(H-1)} \mathrm{d} \underline{s}\right) .
\end{aligned}
$$

In this form one can already recognize the renormalization operator $A_{T}$. Indeed, when $\lim _{T \rightarrow \infty} L_{\infty}(T)=\gamma \in \mathbb{R}_{+}$, then on the right hand side of (4.5) the term $L_{\infty}(T)$ disappears in exchange for parameter $\mu_{0}$ passing into $\mu_{0} \gamma$. Writing the resulting expression in the form of Proposition 2, we have

$$
\begin{aligned}
\lim _{T \rightarrow \infty} \operatorname{cum}\left(\left(A_{T} J_{F}\right)\left(t_{1}\right), \ldots,\right. & \left.\left(A_{T} J_{F}\right)\left(t_{m}\right)\right) \\
& =\mu_{0} \gamma \sigma_{0}^{2 m-2} 2^{2(1-H)}(3-2 H) \Gamma(2-2 H)(m-1) ! \operatorname{sym} \\
&
\end{aligned}
$$

When $F$ is the $\Gamma(3-2 H, 1 / \lambda)$ d.f., then $\gamma=\frac{\lambda^{3-2 H}}{\Gamma(4-2 H)}$, so we get exactly $(2.1)$ with $c_{0}=\mu_{0} \lambda^{3-2 H}$ (see also [3], Sect 3.2, Condition 7).

The main theorem of the paper is the following. 
Theorem 4. Let $\left\{J_{F, i}(t), t \geq 0\right\}, i=1,2, \ldots$, be independent IGSDLG(F) processes and let $\{J(t), t \geq 0\}$ be the LISDLG process with parameters $H, c_{0}=\mu_{0} \Gamma(4-2 H)$ and $\sigma_{0}^{2}$. Then the following weak convergence holds.

$$
\left\{\frac{1}{T} \sum_{i=1}^{\left\lfloor\frac{T^{2(1-H)}}{L \infty(T)}\right\rfloor} J_{F, i}(T t), t \geq 0\right\} \underset{T \rightarrow \infty}{\stackrel{w}{\longrightarrow}}\{J(t), t \geq 0\} \quad \text { in } \mathcal{C}[0, \infty)
$$

$(\lfloor\cdot\rfloor$ denotes the integer part function.)

Proof. The right hand side of (4.7) is the general $m$-fold joint cumulant of process $\{J(t), t \geq 0\}$ (see (2.1)). Because $\lim _{T \rightarrow \infty}\left(T^{2(1-H)} / L_{\infty}(T)\right)=\infty$, Proposition 3 can be applied to show that each joint cumulant of the process on the left hand side of (4.8) converges to the corresponding joint cumulant of process $\{J(t), t \geq 0\}$. Kolmogorov's tightness condition is satisfied too, because

$$
\begin{aligned}
\mathrm{E}\left(\frac{1}{T} \sum_{i=1}^{\left\lfloor\frac{T^{2(1-H)}}{L_{\infty}(T)} J_{F, i}(T t)\right)^{2}}\right. & =\left\lfloor\frac{T^{2(1-H)}}{L_{\infty}(T)}\right\rfloor \operatorname{cum}_{2}\left(\frac{1}{T} J_{F, 1}(T t)\right) \\
& \leq \mu_{0} \sigma_{0}^{2} \int_{0}^{t} \int_{0}^{t} \mathrm{E}_{A}\left(\frac{1}{-2 \alpha} \mathrm{e}^{\alpha T D_{\tau_{0}}(\underline{s})}\right) \frac{T^{2(1-H)}}{L_{\infty}(T)} \mathrm{d} \underline{s} \\
& \leq \mu_{0} \sigma_{0}^{2} \int_{0}^{t} \int_{0}^{t}\left(k_{1} D_{\tau_{0}}(\underline{s})^{2(H-1)+\delta}+k_{2} D_{\tau_{0}}(\underline{s})^{2(H-1)-\delta}\right) \mathrm{d} \underline{s} \\
& =\mu_{0} \sigma_{0}^{2}\left(k_{1} t^{2 H+\delta}+k_{2} t^{2 H-\delta}\right) \\
& <\mu_{0} \sigma_{0}^{2}\left(k_{1}+k_{2}\right) t^{2 H-\delta},
\end{aligned}
$$

where (4.9) follows from (4.6), (4.10) from (4.1), (4.11) is true if we choose $\delta$ so that $0<\delta<2 H-1$ and (4.12) is true for $0<t<1$. The choice $\delta<2 H-1$ ensures also that the power $2 H-\delta>1$, which is needed for the Kolmogorov condition to be satisfied. Thus, the statement of the theorem follows.

Remark 4. The upper limit of the sum in (4.8) can also be written directly in the form

$$
\left\lfloor\frac{T^{2(1-H)}}{L_{\infty}(T)}\right\rfloor=\left\lfloor\frac{1}{T F\left(\frac{1}{T}\right)}\right\rfloor
$$

Remark 5. Formula (4.8) becomes more concrete when $\lim _{T \rightarrow \infty} L_{\infty}(T)=\gamma \in \mathbb{R}_{+}$:

$$
\left\{\frac{1}{(\gamma n)^{\frac{1}{2(1-H)}}} \sum_{i=1}^{n} J_{F, i}\left((\gamma n)^{\frac{1}{2(1-H)}} t\right), t \geq 0\right\} \underset{n \rightarrow \infty}{\stackrel{w}{\longrightarrow}}\{J(t), t \geq 0\} \quad \text { in } \mathcal{C}[0, \infty) .
$$


Acknowledgements. The author thanks the referee for the careful review and his constructive attitude.

\section{REFERENCES}

[1] N.H. Bingham, C.M. Goldie and J.L. Teugels, Regular Variation. Cambridge University Press, Cambridge (1987).

[2] R.L. Dobrushin and P. Major, Non-central limit theorems for nonlinear functionals of Gaussian fields. Z. Wahrsch. Verw. Gebiete 50 (1979) 27-52.

[3] E. Iglói and G. Terdik, Superposition of diffusions with linear generator and its multifractal limit process. ESAIM: PS 7 (2003) 23-86.

[4] A.M. Iksanov and Z.J. Jurek, Shot noise distributions and selfdecomposability. Stoch. Anal. Appl. 21 (2003) 593-609.

[5] P.M. Lee, Infinitely divisible stochastic processes. Z. Wahrsch. Verw. Gebiete 7 (1967) 147-160.

[6] M.S. Taqqu, Convergence of integrated processes of arbitrary Hermite rank. Z. Wahrsch. Verw. Gebiete 50 (1979) 53-83. 